\title{
Artifact-free 3D Reconstruction for Optical Projection Tomography.
}

Jan Michálek ${ }^{1}$, Martin Čapek ${ }^{1}$.

1. Institute of Physiology, Academy of Sciences of the Czech Republic, v.v.i., Vídeňská 1083, Prague 4 -Krč, 14220 Czech Republic.

Optical Projection Tomography (OPT) is a recently developed implementation of computed tomography (CT) techniques at optical frequencies. A series of $2 \mathrm{D}$ optical projections through a sample are generated at varying orientations, from which the 3D structure of the sample can be computationally reconstructed. OPT is especially suitable for samples from about $0.5 \mathrm{~mm}$ to $15 \mathrm{~mm}$ in size, which fills an important "imaging gap" between techniques such as confocal microscopy (useful for smaller samples) and large-sample methods such as fluorescence molecular tomography (FMT), x-ray CT or microscopic magnetic resonance imaging $(\mu \mathrm{MRI})$. OPT can function in both fluorescence and transmission modes.

Fluorescence or absorption images (projections) of the samples are taken over 360 degrees obtained with a fixed rotational angle along the vertical axis, e.g. 0.9 degrees, i.e. 400 projections over 360 degrees.

A standard approach to 3D volume reconstruction of optical projection tomography series uses the well known Filtered Backpojection algorithm (FBP) [1], whose algorithmic foundation is the Radon Transform, first published in 1917. FBP was originally used in other types of tomography, such as X-ray Computed Tomography. Other reconstruction algorithms for OPT were reported rarely [2], and they are not available in standard reconstruction software.

FBP may yield unsatisfactory results in cases where there are large sudden local variations in the brightness of the tomographic projections, which is often the case in OPT. Fig. 1 on the left shows one of a series of 400 fluorescence tomographic projections of a block of the size of approx. $3 \times 3 \times 3 \mathrm{~mm}^{3} \mathrm{cut}^{-}$ from a rat brain, acquired by optical projection tomography (exc/em - 425nm/from $475 \mathrm{~nm}$ ). The brain was stained by Lycopersicon esculentum (tomato) lectin. Prior to acquisition by optical projection tomography (OPT) specimens were cleared using BABB (1 part of benzyl alcohol +2 parts of benzyl benzoate).

In Fig. 1 on the right, one of a total of 503 reconstructed horizontal slices of the specimen is shown. Streak artifacts at the sharp edges of the slice, as well as the halo delimiting the CCD sensor range are well pronounced (darker background is used for better visibility).

For specimens with large regions of almost constant optical density, quality of reconstruction can be greatly improved, and the number of needed projections reduced, when a reconstruction algorithm is used which - apart from aiming at cancelling the error between measured and reconstructed data -minimizes the total variation (TV), i.e. the sum of absolute brightness changes in the reconstructed sections. We used an algorithm based on the theory presented in [3].

Fig. 2 shows that using the total variation minimization algorithm, reconstruction quality is acceptable for as few as 100 projections (i.e. a 3.6 degree step between projections), whereas FBP reconstruction 
deteriorates rapidly. This allows a much higher acquisition turnover, as well as significant disk space savings when repeated acquisitions are inevitable e.g. for OPT settings optimization.

References:

[1] A.Cheddad et al., IEEE Trans Med Imaging. 31 (2012), p.1.

[2] A.Darrell et al., 8th IEEE International Conference on Biolnformatics and Bio Engineering (2008),p. 1.

[3] J. Yang et al. Rice CAAM_TR08-27 (2008).

[4] The authors acknowledge funding from the Czech Republic's public funds provided by Academy of Sciences (AV0Z50110509 and RVO:67985823), Ministry of Education, Youth and Sports (KONTAKT LH13028), and Science foundation of the Czech Republic (13-12412S).
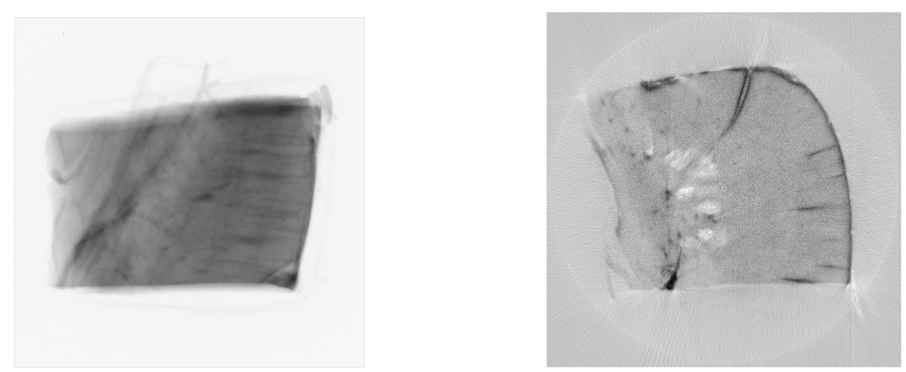

Figure 1. left: fluorescence tomography projection of a block cut from a rat brain, acquired by optical prøjection tomography. right: one section of 3D reconstruction of structures in this block by using the FBP algorithm of the Nrecon software package.

\# projections

400

filtered

backprojection
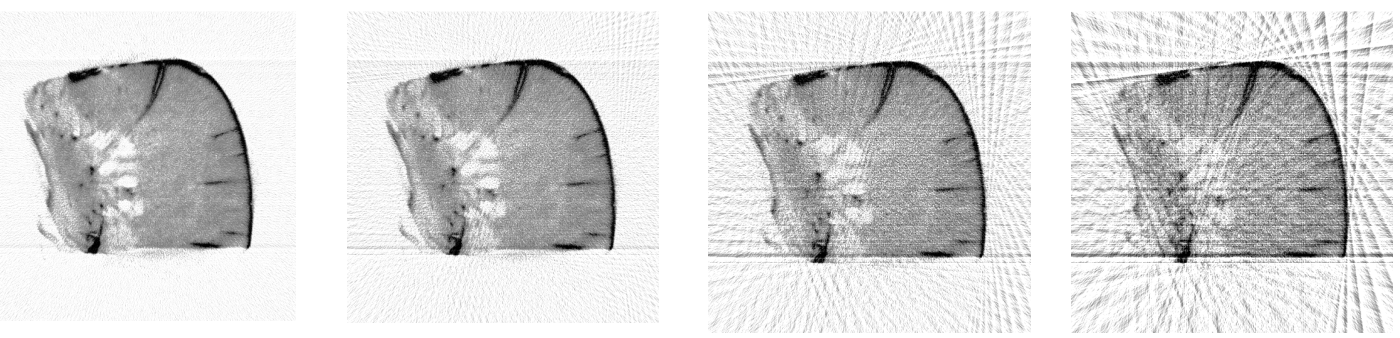

total variation

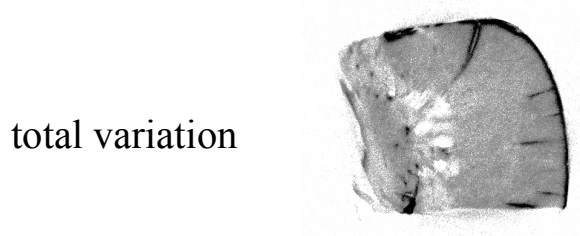

200

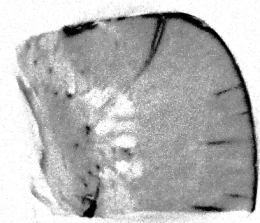

100

50
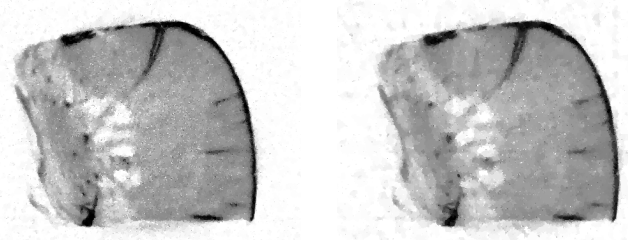

Figure 2. The upper row of images shows how the quality of tomographic reconstruction by the FBP deteriorates fast when a limited number of projections are used for reconstruction. The lower row demonstrates the potential of total variation minimizing algorithms. Reconstruction quality is acceptable down to as few as 100 projections, enabling faster acquisition and lower hard disk requirements. 